ISSN 1870-4069

\title{
Muestreo y reconstrucción de realizaciones de la suma de dos procesos Gaussianos
}

\author{
Vladimir Kazakov, Francisco Mendoza \\ Instituto Politecnico Nacional, ESIME Zacatenco, \\ Departamento de Telecomunicaciones, \\ Ciudad de México, México \\ vkaz41@hotmail.com, fcm2709@gmail.com
}

\begin{abstract}
Resumen. Con base en la regla de la esperanza matemática condicional se investiga el procedimiento de muestreo-reconstrucción para la suma de dos procesos Gaussianos. También se estudian las funciones básicas y la función de error de reconstrucción, cuando el número de muestras y la longitud del intervalo de muestreo son arbitrarios.
\end{abstract}

Palabras clave: suma, procesos Gaussianos, muestreo, reconstrucción.

\section{Sampling and Reconstruction of Realizations of the Sum of Two Gaussian Processes}

\begin{abstract}
Based on the rule of Conditional Mathematical expectation, the sampling-reconstruction procedure is investigated for the sum of two Gaussian processes. The basic functions and the reconstruction error function are also studied, when the number of samples and the length of the sampling interval are arbitrary.
\end{abstract}

Keywords: sum, Gaussian processes, sampling, reconstruction.

\section{Introducción}

La descripción del Procedimiento de Muestreo-Reconstrucción (PMR) de las realizaciones en un proceso aleatorio ha sido investigado durante muchos años (ver por ejemplo dos revisiones [1,2]). Según el teorema de Balakrisnhan (TB) [3] se puede reconstruir cualquier realización de un proceso aleatorio estacionario con un espectro de potencia restringido a una frecuencia $\omega_{b}$ usando la siguiente expresión:

$$
x(t)=\lim _{N \rightarrow \infty} \sum_{i=-N}^{N}\left(x\left(T_{i}\right)\right) \frac{\operatorname{sen} \omega_{b}\left(t-i T_{b}\right)}{\omega_{b}\left(t-i T_{b}\right)},
$$

donde $x\left(T_{i}\right)$ la muestra de una realización en el instante $T_{i} ; \Delta T$ es un intervalo de muestreo periódico entre las muestras vecinas:

$$
T_{b}=T_{i}-T_{i-1}=\frac{\pi}{\omega_{b}} .
$$


Enfatizamos que en TB el error es cero si y solo si el número de las muestras es infinito . En [5,6] fue probado que TB es válido solamente para procesos Gaussianos.

El algoritmo (1) se caracteriza por tener un error de reconstrucción igual a cero para todos los procesos independientemente de su función de densidad de probabilidad [4]. Introducimos la función básica $b_{i}(t)$.

Como se ve en (1) todas la muestras tienen la misma función básica del tipo $\frac{\operatorname{sen}(x)}{x}$ :

$$
b_{i}(t)=\frac{\operatorname{sen} \omega_{b}\left(t-i T_{b}\right)}{\omega_{b}\left(t-i T_{b}\right)} .
$$

Notamos, que el algoritmo (1) y la función básica (3) ignoran tales características de gran importancia como: la función de densidad de probabilidad $(f d p)$, y la función de covarianza del proceso.

El modelo matemático de procesos aleatorios con espectro restringido es citado con mucha frecuencia en la literatura. Pero TB no da ninguna información acerca de la de la influencia para PMR cuando el número $N$ de muestras son arbitrarias. El artículo presente da la claridad dentro de dichos problemas, porque para cada variante se obtienen dos características más importantes para cada PMR: la función básica y del error de reconstrucción. Estos resultados pueden ser útiles para los investigadores de los sistemas de comunicación con mensajes gaussianos con espectros rectangulares.

La meta del artículo presente es investigar el problema PMR de las realizaciones de procesos Gaussianos con un espectro rectangular, centrado en el origen y al encontrarse desplazado, cuando $\Delta T$ y $N$ son arbitrarios.El conocimiento de la forma del espectro nos da la posibilidad de conocer la función de covarianza del proceso dado. Para cumplir este análisis usamos la metodología de la regla de esperanza matemática condicional [7]. La aplicación de dicha regla para la descripción de PMR de realizaciones de procesos aleatorios de varios tipos, fue descrita en algunas publicaciones [5,6], por mencionar algunos. En la investigación de PMR hay dos características principales: 1) la función óptima de reconstrucción; 2) la función mínima del error de la reconstrucción.

\section{La regla de la esperanza matemática condicional en PMR de realizaciones gaussianas}

Cada proceso gaussiano no estacionario esta descrito con tres características principales: la esperanza matemática $m(t)$, la varianza $\sigma^{2}(t)$ y la función de covarianza $R\left(t_{1}, t_{2}\right)$. Elegimos una realización del proceso y fijamos una multitud de las muestras $X, T=\left\{X\left(T_{1}\right), X\left(T_{2}\right), \ldots, x\left(T_{N}\right)\right\}$. En esta multitud el número de muestras $N$ y la locación de la muestras $T=\left\{T_{1}, T_{2}, \ldots, T_{N}\right\}$ son arbitrarios. Usando $X, T$ las características a priori $m(t), \sigma^{2}(t), R\left(t_{1}, t_{2}\right)$ se puede obtener las características del proceso condcional $x(t) \mid X, T=\tilde{x}(t)$, es decir, la esperanza matemática condicional $\langle x(t) \mid X, T\rangle=\tilde{m}(t)$, y la varianza condicional $\langle[\tilde{x}(t)-$ $\tilde{m}(t)]^{2}|X, T\rangle=\tilde{\sigma}^{2}(t)$. 
La función $\tilde{m}(t)$ es la función de reconstrución de la realización muestreada y la función $\tilde{\sigma^{2}}(t)$ es la función de error de reconstrucción. La función $\tilde{m}(t)$ es la mejor estimación de la realización en el instante de tiempo actual $t$ y la función $\tilde{\sigma}^{2}(t)$ es el error mínimo de reconstrucción de dicha realización en el mismo instante de tiempo $t$. Las características principales de PMR son $\tilde{m}(t)$ y $\tilde{\sigma}^{2}(t)$ pueden ser descritas según [8]:

Considerando el caso estacionario para $\tilde{m}(t)$ y $\tilde{\sigma}^{2}(t) ; m(t)=m=0, \sigma^{2}(t)=$ $\sigma=1, R\left(t_{1}, t_{2}\right)=R\left(t_{1}-t_{2}\right)$, tienen las formulas siguientes:

$$
\begin{gathered}
\tilde{m}(t)=\sum_{i=1}^{N} \sum_{j=1}^{N} R\left(t-T_{i}\right) a_{i j} x\left(T_{j}\right), \\
\tilde{\sigma}^{2}(t)=1-\sum_{i=1}^{N} \sum_{j=1}^{N} R\left(t-T_{i}\right) a_{i j} R\left(T_{j}-t\right), \\
\left\|R\left(T_{i}-T_{j}\right)\right\|=\left\|\begin{array}{l}
R\left(T_{1}-T_{1}\right) \ldots R\left(T_{1}-T_{N}\right) \\
\ldots\left(T_{N}-T_{1}\right) \ldots R\left(T_{N}-T_{N}\right)
\end{array}\right\|, \\
\left\|a_{i j}\right\|=\left\|R\left(T_{i}-T_{j}\right)\right\| .
\end{gathered}
$$

La expresión (4) se reescribe en forma simplificada

$$
\tilde{m}(t)=\sum_{j=1}^{N} x\left(T_{j}\right) b_{j}(t)
$$

aquí es una función básica determinada por la formula

$$
b_{j}(t)=\sum_{j=1}^{N} R\left(t-T_{i}\right) a_{i j} .
$$

Cada muestra tiene su propia función $b_{j}(t)$.

Considerese la suma de dos procesos aleatorios gaussianos estacionarios $z(t)$ en la salida $y_{1}(t)$ y $y_{2}(t)$ de dos transformaciones lineales, caracterizados por su respuesta al impulso $h_{1}(t) y h_{2}(t)$. Los cuales tienen a la entrada $x(t)$ ruido blanco. Por tanto, la suma $z(t)$ de procesos aleatorios será otro proceso aleatorio gaussiano. Donde su función de covarianza normalizada $r(\tau)$ esta determinada por $(10)$ :

$$
r(t)=\frac{R_{y_{1}}(\tau)+R_{y_{2}}(\tau)+2 R_{12}(\tau)}{R_{y_{1}}(0)+R_{y_{2}}(0)+2 R_{12}(0)},
$$

donde $R_{y_{1}}(\tau)$ es la función de covarianza del proceso aleatorio $y_{1}(t), R_{y_{2}}(\tau)$ es la función de covarianza del proceso aleatorio $y_{2}(t)$, y $R_{12}(\tau)$ es la función de covarianza mutua entre los procesos $y_{1}(t)$ y $y_{2}(t)$. Se utilizan las ecuaciones (4)-(9) para definir las caracteristicas condiciones en la descripción del PMR. 


\section{Resultados obtenidos del PMR de la suma de procesos aleatorios para diferentes filtros lineales}

A continuación se muestran los resultados para diferentes casos: 1) la suma dos procesos aleatorios cuando ambos procesos aletorios son transformados por un filtro $\mathrm{RC}$ de una etapa con diferentes constantes de tiempo; 2) la suma de un proceso aleatorio transformado por un filtro $\mathrm{RC}$ de dos etapas y otro proceso por un filtro RC de una etapa, con misma constante de tiempo; .

\subsection{Suma de dos procesos aleatorios transformados por dos diferentes filtros RC de una etapa}

De manera general, a la transformación lineal de primer orden de procesos gaussianos se obtienen procesos gaussiano markoviano, esto significa, que entre las variables aleatorias solo existira una dependencia inmedianta entre el vecino que represente el presente y el futuro. Por esta razon, la reconstrucción de un proceso aleatorio gaussiano markoviano es el mas fácil, para estudiar la influencia de las constantes de tiempo sobre el PMR.

Para usar la regla de la esperanza matemática condicional en el PMR es necesario conocer la función de covarianza. En nuestro caso la función de covarianza $r(\tau)$ de la suma de procesos aleatorios. Por la formula(10) se debe conocer la función de covarianza de cada proceso aleatorio a la salida de cada circuito RC de una etapa, es decir, $R_{y_{1}}(t)$ y $R_{y_{2}}(t)$, en (11) y (12):

$$
\begin{aligned}
& R_{y_{1}}(t)=\exp (-\alpha|\tau|), \\
& R_{y_{2}}(t)=\exp (-\beta|\tau|) .
\end{aligned}
$$

También, la función de covarianza mutua $R_{12}$ entre el proceso $y_{1}(t)$ y $y_{2}(t)$, que se define en (13):

$$
R_{12}(\tau)=\frac{\alpha \beta}{\alpha+\beta}\left\{\begin{array}{l}
\exp (-\alpha|\tau|) \text { si } \tau>0 \\
\exp (-\beta|\tau|) \text { si } \tau<0
\end{array}\right.
$$

Realizando las sustituciones correspondientes de las formulas (11), (12) y (13) en (10). Se obtine la función de covarianza de la suma de los procesos, caracterizado por (11) y (12), a la salida de filtros RC de primera etapa. En Fig.1. se muestran las diferentes funciones de covarianza $R_{y_{1}}(\tau), R_{y_{2}}(\tau), R_{12}(\tau)$ y $r(\tau) \operatorname{con} \alpha=1$ y $\beta=0,1$.

A continuación se describe el PMR para la suma de dos procesos aleatorios, utilizando las formulas (4)-(9), estudiando la influencia de la constante de tiempo.

En Fig. 2. se muestran las funciones básicas cuando $\alpha=1$ y $\beta=0,1 ; 0,5 ; 0,9$ con un número de muestras $N=2$ y un intervalo de muestreo $\delta T=0,2$.

Enfatizamos algunas características generales de las funciones básicas: 1)cada muestra tiene su función básica; 2)en cada instante de muestreo, solamente una función tiene el valor de uno, mientras todas las demas funciones son cero en el 


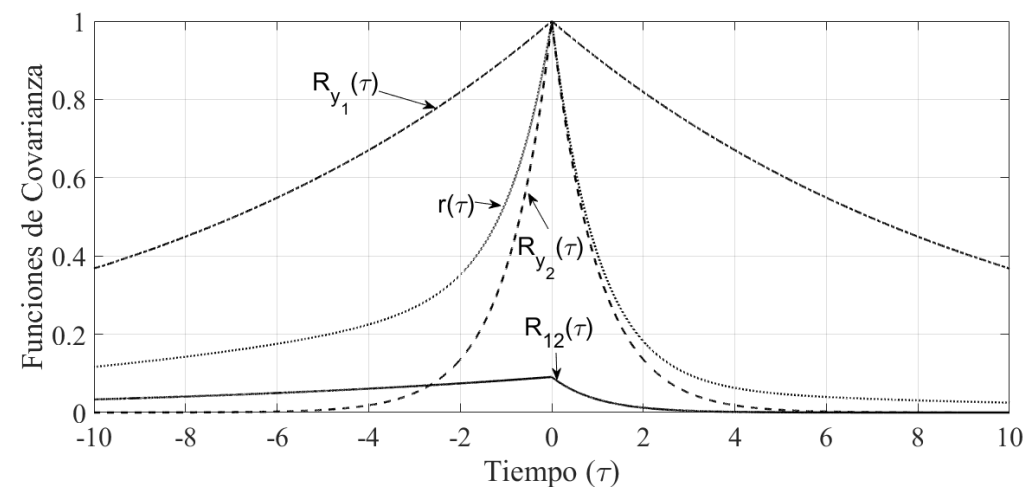

Fig. 1. Funciones de Covarianza $\alpha=1$ y $\beta=0,1$.

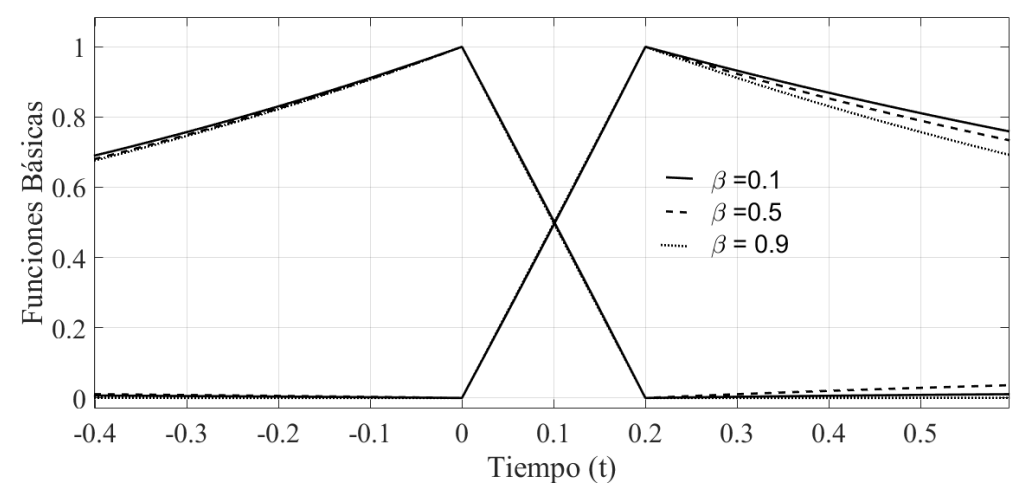

Fig. 2. Funciones básicas a diferentes anchos de banda

mismo intante de muestreo, es decir, cada función básica es orto-normal entre las demas. De manera particular, se observa: 1 ) en la región $T_{1}<t<T_{N}$ (región de interpolación) el comportamiento de reconstrucción es igual a lineal, en los tres casos; 2) en las regiones de extrapolación $\left(t>T_{N}\right)$ y retropolación $\left(t<T_{1}\right)$ presentan diferentes pendientes con la variación de la constante de tiempo de uno de los filtro, fisicamente el cambio en la constante del tiempo no afecta directamente la eficiencia de reconstrucción.

Por último consideremos, la calidad de reconstrucción del PMR utilizando los mismos parametros, es decir, $\alpha=1$ y $\beta=0,1 ; 0,5 ; 0,9$ con un número de muestras $N=2$ y un intervalo de muestreo $\delta T=0,2$.

De manera general, la función de error de reconstrucción tiene las características: 1) a mayor longitud del intervalo de muestreo $\Delta T$ mayor el error de reconstrucción; 2) entre mayor cantidad de muestras menor será el error de reconstrucción. En Fig. 3. se observa que las funciones de error de reconstrucción 


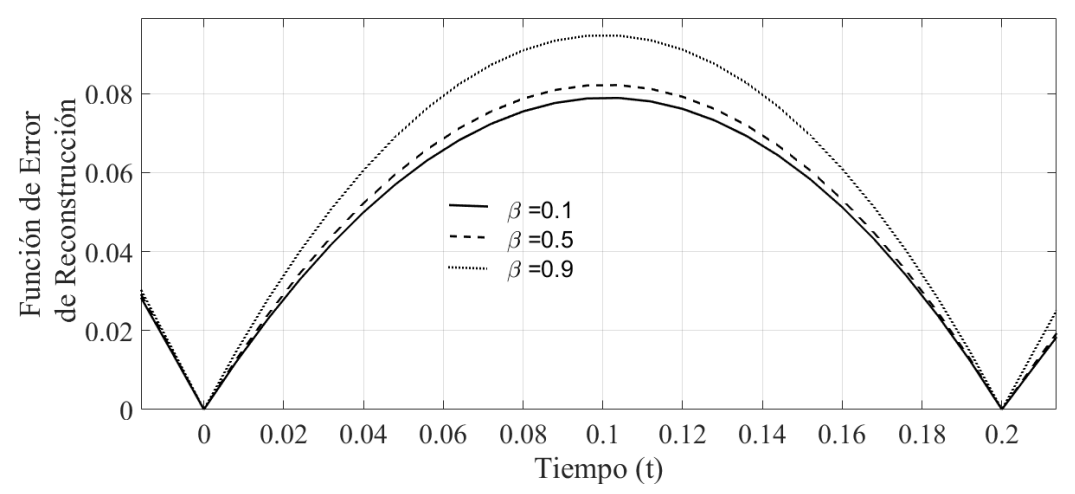

Fig. 3. Funciones básicas a diferentes anchos de banda

no varia significativamente, aun cuando se compara el caso $\beta=0,1$ y $\beta=0,9$ Esto quiere decir, que en el PMR no existe una influencia determinante por la variación las constantes de tiempo, sino del sistema al cual esta sometido la transformación de los procesos a sumar.

\subsection{Suma de dos procesos aleatorios transformados por un filtro RC de dos etapas y una etapa}

A diferencia del caso anterior que se estudiaba la influencia del ancho de banda en PMR de la suma de procesos aleatorios. Ahora se investigará la influencia de la estructura de los sistemas sobre el PMR, cuando el ancho de banda es igual para dos sistemas diferentes. Por tanto, se comenzará definiendo la funciones de covarianza en la salida de los filtros RC de primera y segunda etapa, cuando a su entrada existe ruido blanco, en (14) y (15) respectivamente:

$$
\begin{gathered}
R_{y_{1}}(\tau)=\exp (-\alpha|\tau|), \\
R_{y_{1}}(\tau)=(1+\alpha|\tau|) \exp (-\alpha|\tau|) .
\end{gathered}
$$

Se obtiene la función de covarianza mutua entre los procesos $y_{1}(t)$ y $y_{2}(t)$, en $(16)$ :

$$
R_{12}(\tau)= \begin{cases}\frac{(1+2 \alpha \tau) \exp (-\alpha|\tau|)}{\sqrt{2}} & \text { si } \tau>0 \\ \frac{\exp (-\beta|\tau|)}{\sqrt{2}} & \text { si } \tau<0\end{cases}
$$

De (10) se obtine la función de covarianza de la suma de los procesos procesos aleatorios $y_{1}(t)$ y $y_{2}(t)$. En Fig.4 se muestran las diferentes funciones de covarianza definidas en (11)-(13).

Usando las ecuaciones (4)-(9) se ilustrará las caracteristicas condicionales para el estudio del PMR. En Fig. 5, se muestran las funciones básicas del PMR 


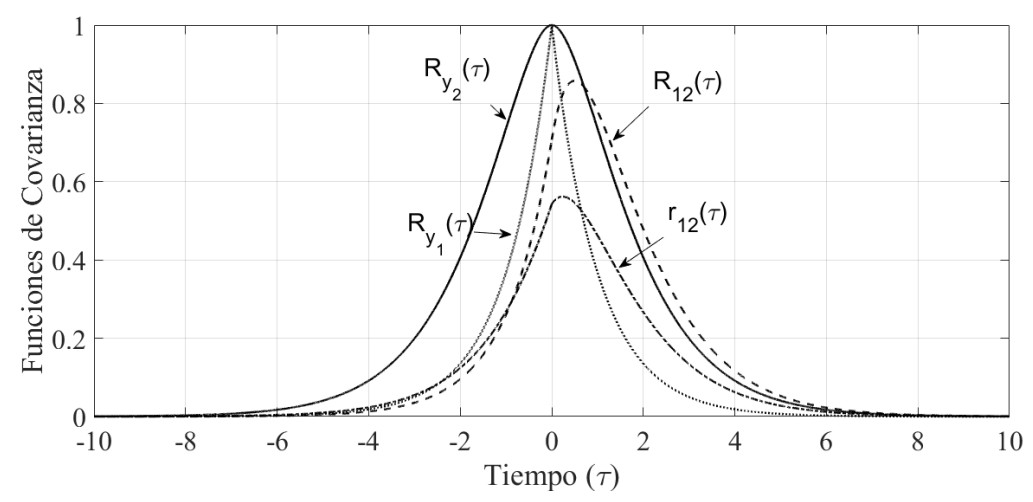

Fig. 4. Funciones de covarianza

cuando con una constante de tiempo $\alpha=1 ; 0,5$ y un intervalo de muestreo $\Delta T=0,2$.

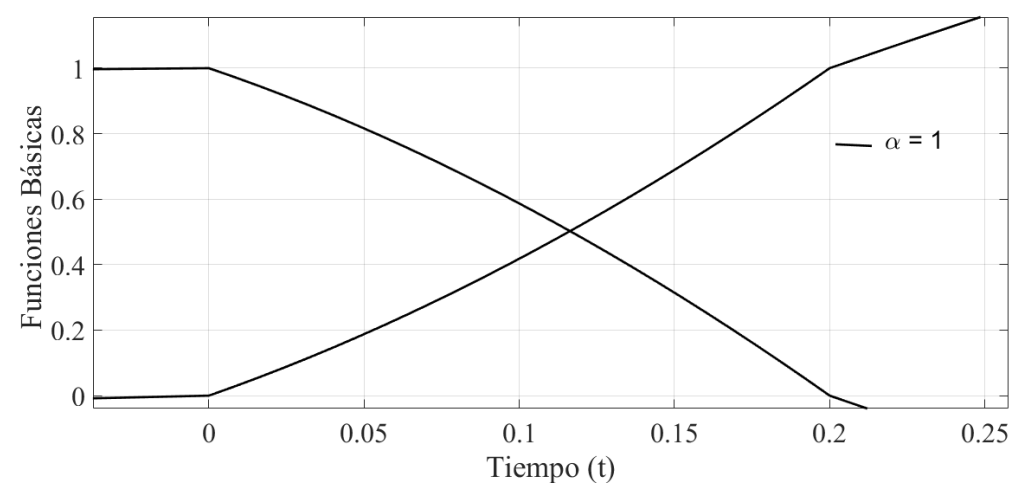

Fig. 5. Funciones básicas

En Fig. 5, se observa que la tendencia en la región de interpolación no es lineal, a diferencia de Fig.2. Esto es resultado de la interacción entre los filtros RC de una y dos etapas, dado que este último el proceso en su salida será de tipo no markoviano a diferencia de los casos anteriores. Por último consideremos la función de error de reconstrucción, con los mismos parametros al caso anterior.

En Fig. 6, se muestran la función de error de reconstrucción. Al compararse cuantitativamente con la Fig.3, se observa una mejora significativa en la calidad de la reconstrucción al presentar la influencia de un filtro de dos etapas, pues este último será un proceso no markoviano, es decir, que existe una relación de dependencia entre todas sus variables aleatorias. De manera particular, significa 


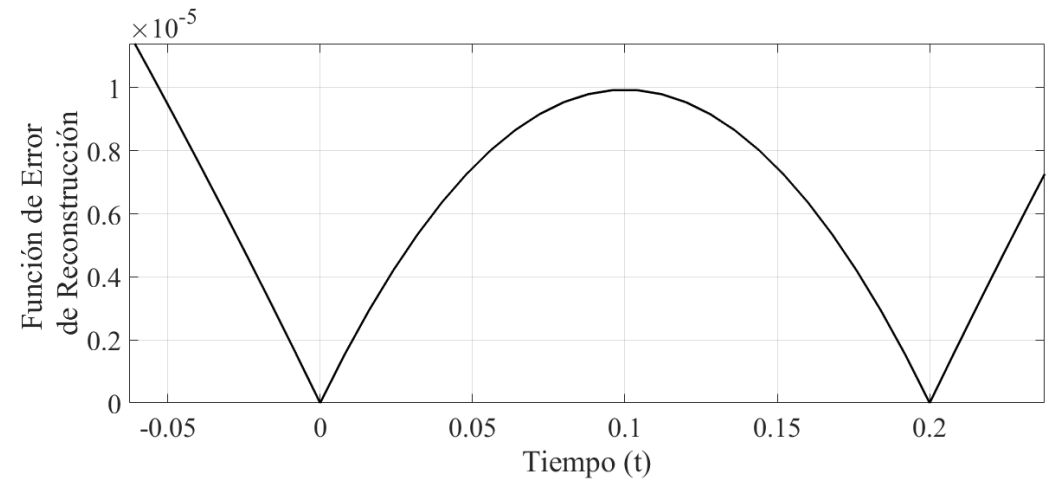

Fig. 6. Funciones básicas

que la dependencia estadística existente en un proceso juega un papel importante para reconstrucción de dicho proceso.

\section{Conclusiones}

Utilizando la regla de la esperanza matemática condicional, como metodología de la investigación, del cálculo de errores de reconstrucción cuando el número de muestras es limitado y cuando los intervalos de muestreo son arbitrarios, en un proceso Gaussiano como resultado de la suma de dos procesos gaussianos transformados linealmente, se observa que a mayor dependencia estadística entre las variables aleatorias (en los instantes de muestreo) que componen el conjunto de muestras la reconstrucción será de mayor calidad. Por tanto, el tipo de transformación lineal influyen en el PMR. También se observa que la constante de tiempo no afecta de manera significativa la calidad de la reconstrucción.

Es bien sabido que el área de procesamiento de señales de voz, el procedimiento de Muestreo-Reconstrucción es una etapa importante para el tratamiento de este tipo de señales. La aplicacion de la REMC en el PMR de señales de voz es una alternativa a investigar en la identificación de fonemas [8].

\section{Referencias}

1. Jerry, A.: The Shannon sampling theorem: Its various extensions and applications. A tutorial review. Proc. IEEE, vol. 65, pp. 1565-1596 (1977)

2. Jerry, A.: Bibliographic List. In: Advanced Topics in Shannon sampling and interpolation theory. Springer, NY (1992)

3. Balakrishnan, A.: A Note on the Sampling Principle for Continuous Signals. IRE Trans. On information theory, Vol. IT-3, pp. 143-146 (1957)

4. Balakrishnan, A.: On the Problem of Time Jitter in Sampling. IRE Trans. On information theory, Vol. IT-3, pp. 226-236 (1962) 
5. Kazakov, V.: The sampling-reconstruction procedure with a limited number of samples of stochastic processes and fields on the basis of the conditional mean rule. Electromagnetic waves and electronic systems, Vol.10, Num. 1-2, pp. 98-116 (2005)

6. Kazakov, V.: Sampling-Reconstruction procedures of Gaussian process realizations. In: Probability: interpretation, theory and applications. Y. Shmaliy (ed.), Nova science publisher inc., USA, NY, pp.269-297 (2012)

7. Pfeiffer, P.: Probability for applications. Springer Verlag (1990)

8. Proakis, J., Manolakis, D.: Digital signal processing: Principles, algorithms, and applications. Springer Verlag (1990) 